\title{
Use of a supercontinuum white light in evaluating the spectral sensitivity of the pupil light reflex
}

Catherine Chin, Lasse Leick, Adrian Podoleanu, Gurprit S. Lall

Catherine Chin, Lasse Leick, Adrian Podoleanu, Gurprit S. Lall, "Use of a supercontinuum white light in evaluating the spectral sensitivity of the pupil light reflex," Proc. SPIE 10591, 2nd Canterbury Conference on OCT with Emphasis on Broadband Optical Sources, 1059105 (5 March 2018); doi: $10.1117 / 12.2286064$

Event: Second Canterbury Conference on Optical Coherence Tomography, 2017, Canterbury, United Kingdom 


\title{
Use of a Super continuum white light in evaluating the spectral sensitivity of the pupil light reflex
}

\author{
Catherine Chin ${ }^{1,2,3}$, Lasse Leick ${ }^{3}$, Adrian Podoleanu ${ }^{1}$, Gurprit S Lall ${ }^{2}$ \\ ${ }^{1}$ Applied Optics Group, University of Kent, Canterbury CT2 7NH, UK \\ ${ }^{2}$ Medway School of Pharmacy, University of Kent, Chatham ME4 4TB, UK \\ ${ }^{3}$ NKT Photonics A/S, Blokken 84, Birkerød, DK-3460, Denmark \\ *Corresponding author: G.Lall@kent.ac.uk
}

\begin{abstract}
We assessed the spectral sensitivity of the pupillary light reflex in mice using a high power super continuum white light (SCWL) source in a dual wavelength configuration. This novel approach was compared to data collected from a more traditional setup using a Xenon arc lamp fitted with monochromatic interference filters. Irradiance response curves were constructed using both systems, with the added benefit of a two-wavelength, equivalent power, output using the SCWL. The variables applied to the light source were intensity, wavelength and stimulus duration through which the physiological output measured was the minimum pupil size attained under such conditions. We show that by implementing the SCWL as our novel stimulus we were able to dramatically increase the physiological usefulness of our pupillometry system.
\end{abstract}

Keywords: Pupil, Mouse, Super continuum, dual wavelength

\section{INTRODUCTION}

The mammalian eye is an essential component of the central nervous systems' sensory complement. It is primarily, responsible for generating a visual representation of our environment. This is possible through the decoding of light by a structure termed the retina, which contains complex neuronal structures capable of detecting the spectral composition of a light source and converting this into an electrical impulse [1]. The retina, itself, coats the back of the inner eyeball and forms optic nerves that feed the brain; ultimately terminating at the occipital lobe. Its composition consists of a number of relay networks, which contain specialised cells capable of converting light, photons, into electrical signals. These cells are termed photoreceptors [2].

The mammalian visual system consists of three types of photoreceptor defined by their spectral sensitivity to light. Rods and cones are typically referred to as the 'classic' photoreceptors and are responsible for creating a visual image of our environment $[1,3,4]$. A third class of photoreceptor has been identified in the retinal ganglion cell layer of the retina, with the sole role of encoding ambient light for non-image forming (NIF) visual responses e.g. pupillary light reflex, circadian entrainment- impacting behavioural/ physiological responses $[2,5,6]$. However, establishing the individual contribution of each photoreceptor class to NIF events remains problematic due to the overlap in spectral sensitivity across these cell types (Fig. 1). Traditionally, studies have used murine transgenic models, which either ablate or disable function of a particular photoreceptor class [1]. However, a limitation of such an approach is the likely probability of alterations in physiological function and structural reorganisation of the retina [7].

The pupillary light reflex (PLR) is an autonomic response to bright light. It is well established that the degree of iris constriction is relative to the intensity of the source light being presented to the eye [8]. Foremost, this reflex acts to protect the retina from damage by bright light. However, we can also use this reflex to understand how light is decoded by the retina and used to determine a physiological output. Through manipulation of the light source, for both intensity and wavelength, it is possible to assess/ allude to the contribution of each receptor class to the PLR [1]. Clinically, the PLR has shown promise in the assessment of brainstem function and in the management of pain, through the impact of pupil sensitivity to opioids $[9,10]$.

2nd Canterbury Conference on OCT with Emphasis on Broadband Optical Sources, edited by Adrian Podoleanu, Ole Bang, Proc. of SPIE Vol. 10591, 1059105

(C) 2018 SPIE · CCC code: 1605-7422/18/\$18 · doi: 10.1117/12.2286064 
Traditionally, the PLR setup has utilised a powerful xenon arc light source with wavelength and intensity all regulated by chromatic/ neutral density filters [4]. This setup is ideal for looking the change in pupil constriction to single intensities at particular wavelengths, be it monochromic or bandpass in nature; however lacks the ability to look at responses to dynamically changing light stimuli.

In this study, we propose the use of a broadband super continuum white-light (SCWL) source to generate a dual light stimulus of equivalent power. We will use this design to show the ability of a two-channel setup in demonstrating the retinal sensitivity to light. There is great potential in this novel use of a SCWL in both providing better understanding of photoreceptor contribution to physiological outputs as well as increasing the resolution of this technique to assist in clinical diagnosis and healthcare.

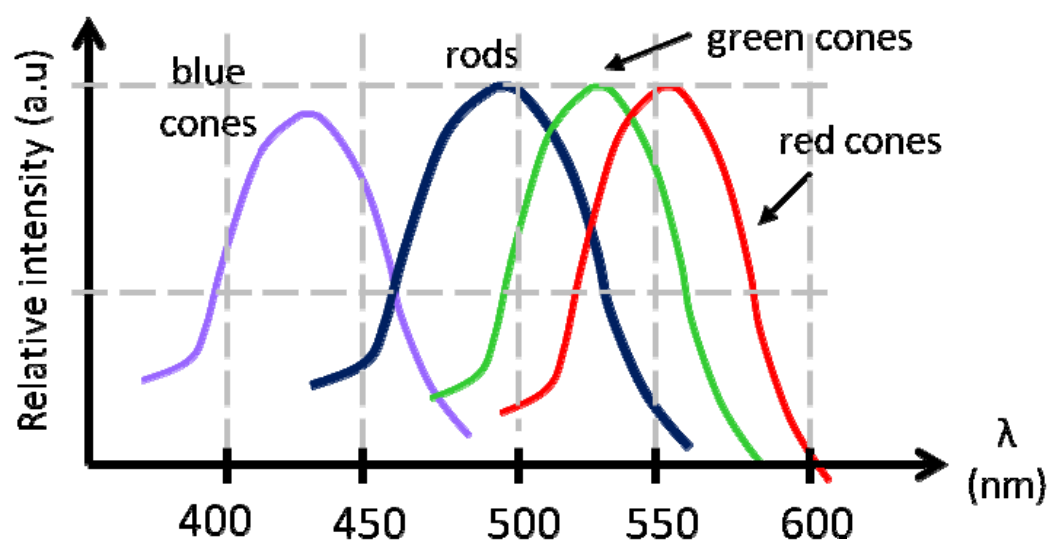

Fig. 1. Spectral sensitivity profiles of the classic retinal photoreceptors. There is a clear distinction in sensitivity profiles of each receptor, however there is also great overlap. In an intact retina, it is extremely difficult to tease out the contribution of each photoreceptor to a physiological or behavioural response. Margins

\section{Methods}

\subsection{Animals}

Experimental procedures were carried out under licence by the Home Office (UK) in accordance with the Animals (Scientific Procedures) Act (1986) and following ethical approval from the University of Kent ethics board. Adult C57BL/6J mice (50-80 days old, $\mathrm{n}=20)$ were maintained under a 12:12 light -dark (LD) cycle, room temperature $22 \pm 2$ ${ }^{\circ} \mathrm{C}$. All efforts were made to minimise the number of animals used and their suffering.

\subsection{Pupillometry}

Pupillometry was conducted as previously described by Lucas et al., 2003 [8]. Briefly, animals were stably entrained to a $12 \mathrm{hr}$ : $12 \mathrm{hr}$ LD cycle (white LED source, 1000 lux) and recordings were restricted to between 4 and $7 \mathrm{hr}$ after lights on. All experiments were preceded by $1 \mathrm{hr}$ of dark adaptation (Fig. 2). Pupillary responses were elicited through a Ganzfeld integrating sphere applied to one eye, previously dilated with $0.1 \%$ atropine, allowing consensual pupil constriction from the other eye to be recorded with a CCD camera. Light sources consisted of either a Xenon arc source, filtered with neutral density and monochromatic interference filters, half bandwidth $\leq 10 \mathrm{~nm}$ (Fig. 4) or a dual laser configuration (Fig. 5 ) with a broadband SCWL source, and two computer controlled wavelength selection modules. Stimulus protocols were programed and run via the associated NKT controller computer software (Fig. 2). 


\subsection{Image capture and analysis}

Pupil recordings lasted for 63 seconds in all experiments. Images were captured at a frequency of six per second. The first three seconds were used to record fully dilated dark-adapted pupils. For the experiments using they Xeon arc, images were captured for 60 seconds with the stimulus set to a $480 \mathrm{~nm}$ light. However, under the SCWL, images were taken for 15 second under a $560 \mathrm{~nm}$ light and then the addition of a $480 \mathrm{~nm}$ light for 45 seconds ensued.

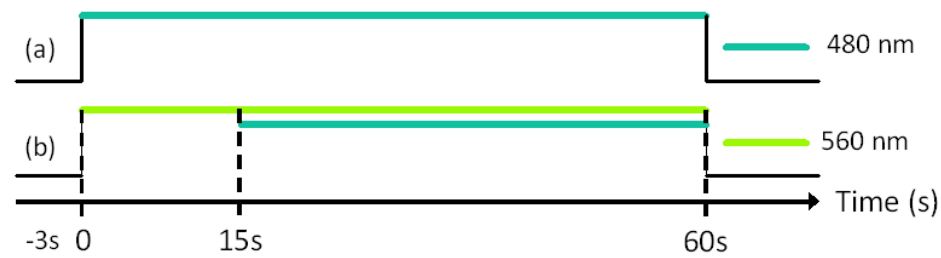

Fig. 2. Stimulus protocols for (a) Xenon arc consisted of a single $480 \mathrm{~nm}$ light pulse for $60 \mathrm{~s}$, and through the SCWL (b) a dual stimulus was generated with $560 \mathrm{~nm}$ for $60 \mathrm{~s}$ and a $480 \mathrm{~nm}$ signal for the last $45 \mathrm{~s}$.

Pupil areas were measured using ImageJ software (v1.50, NIH, open source). Collected images were imported into ImageJ as TIFF files (8-bit). The ellipse tool was used to highlight the circumference of the pupil and the area (pixels ${ }^{2}$ ) computed (Fig. 3).

(a)
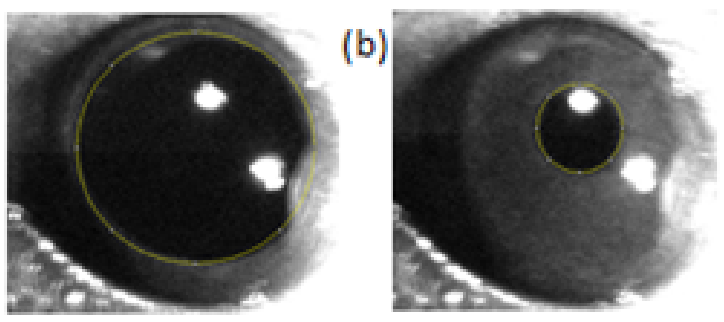

Fig. 3. Pupil area was measured by drawing ellipses around the circumference of the pupil in dark adapted, dilated (a) and constricted (b) eyes.

Minimum pupil sizes were calculated by obtaining the smallest pupil size attained under any given light stimulus and presented relative to the dark-adapted pupil areas of individual animals. In detail, the normalised minimum pupil area $\left(\mathrm{A}_{\mathrm{Npupil}}\right)$ is calculated by dividing the smallest pupil area achieved through the stimulus duration $\left(\mathrm{N}_{\min }\right)$ by the largest dark-adapted pupil ( $\mathrm{N}_{\text {ref }}$ ) pre-stimulus (Eqn. 1).

$$
A_{\text {Npupil }}=\frac{N_{\min }}{N_{\text {ref }}}
$$

Statistical analysis: Nonlinear regression (curve fit) analysis was performed on irradiance response curves. Significance was assigned using the extra sum-of-squares F test set to $p<0.05$. All analysis was undertaken using Graphpad Prism, V.6 for Macintosh, Graphpad Software, La Jolla CA USA.

\subsection{Apparatus}

(i) Xenon Arc: The traditional, single light source, pupillometry apparatus typically consists of a full spectrum xenon arc lamp fitted with a filter holder and shutter. A light pipe was used to feed the integrating sphere, where the light stimulus was presented to the mydriatic containing eye (Fig. 4). 


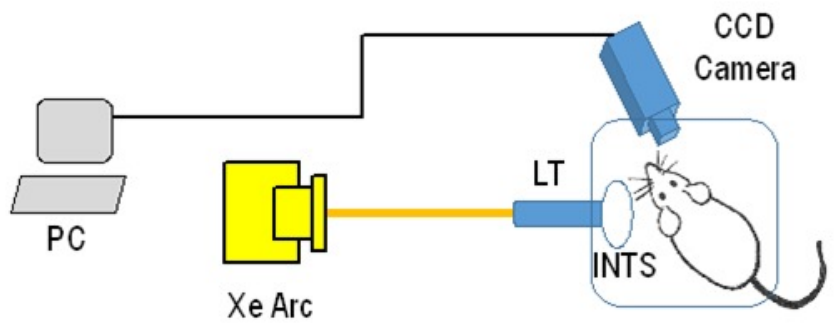

Fig. 4. Schematic of a single Xeon (Xe) arc light source used to provide a stimulus, through a light tube (LT) into an integrating sphere (INTS), for the recording of a consensual pupillary constriction via a charge-coupled device (CCD) camera.

(ii) Super continuum white light source: A broadband SCWL source (SuperK Extreme EXR20, 500-2400 nm, NKT Photonics) provided a dual light configuration capable of generating high power light at two different wavelengths. The output of the SCWL was fed into two wavelength tuning units, a visible/NIR multi-line selector (SuperK VARIA, 500$840 \mathrm{~nm}$, NKT Photonics) to generate $560 \mathrm{~nm} \pm 10 \mathrm{~nm}$ light, and a UV extension module (EXTEND-UV, 350-480 nm, NKT Photonics) to produce light at a bandwidth of $480 \mathrm{~nm} \pm 10 \mathrm{~nm}$. Wavelength selection was made possible through a single interface user software developed by NKT on a PC running Microsoft Windows.

The $560 \mathrm{~nm}$ light from Varia was reflected off a gold mirror (M) and combined with $480 \mathrm{~nm}$ light from Extend-UV using a long pass dichroic mirror (DM, DMLP505, Thorlabs). The combined beams were then coupled into one end of the silica light tube (LT) with the other providing the input port for the integrating sphere, INTS (IS200-4, Thorlabs). The effective light power was measured using a digital handheld optical power meter (PM100D, Thorlabs) equipped with a Silicon diode sensor (S120C, Thorlabs) from the stimuli presenting port ( $\operatorname{area}=0.5 \mathrm{~cm} 2)$ (Table 1). Each of the two wavelength beams had a $1 \mathrm{~mW}$ initial power measured at the output port of the INTS.

Absorptive neutral density filters (part numbers NE10A to NE40A, Thorlabs) were used to attenuate power by a factor of 10. The effective quantum flux densities (in $\log 10$ eff photons $/ \mathrm{cm} 2 / \mathrm{s}$ ) of each stimulus was estimated by integrating the spectral power density of illuminating wavelengths $(470-480 \mathrm{~nm}$ and $555-565 \mathrm{~nm})$ using Eqn. 2. The corresponding power values are shown in Table 1.

$$
N_{p}=\frac{E_{c}}{E_{p}}=\frac{E \cdot \lambda \cdot 10^{-9}}{h \cdot c}=E \cdot \lambda \cdot 5.03 \times 10^{15}
$$

Where $\mathrm{E}=\mathrm{P} / \mathrm{ds}$ is the illuminating energy per unit area, $\mathrm{P}$ is the power metered intensity in $\mathrm{mW}$ and ds is the illuminating beam spot in $\mathrm{cm}^{2}$.

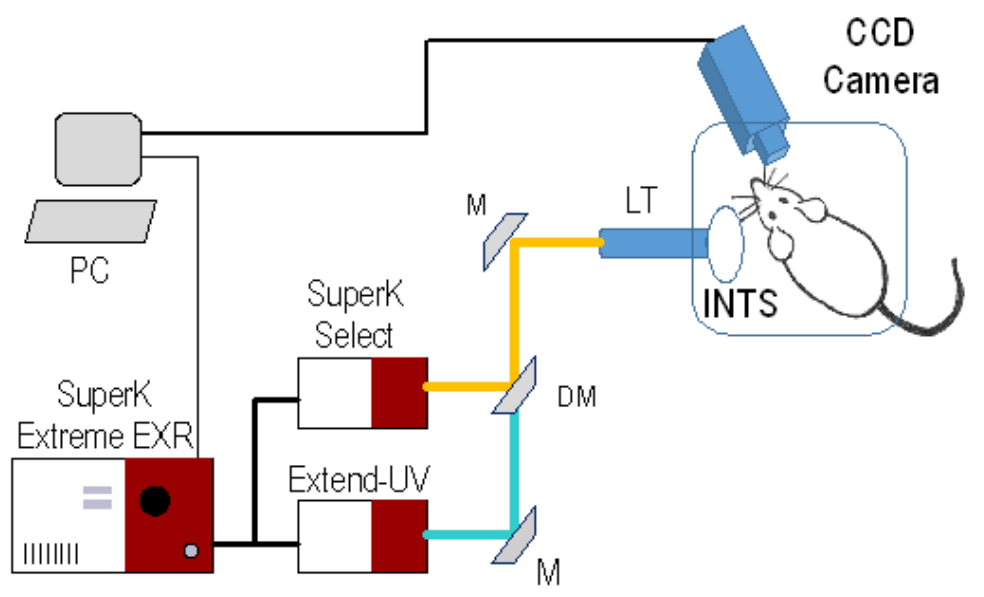

Fig. 5. Schematic of the dual stimulus SCWL light source. Light was generated by the SuperK Extreme EXR and filtered through Extended UV (480 $\mathrm{nm})$ and SuperK Select $(560 \mathrm{~nm})$ modules. Mirrors $(\mathrm{M})$ and dichroic mirrors $(\mathrm{DM})$ were used to direct light into a silica light tube (LT) attached to an integrating sphere (INTS). 


\section{Results}

The initial set of experiments was preformed using the traditional Xenon arc light source. Minimal pupil sizes were measured following exposure to a single intensity of $480 \mathrm{~nm}$ light. An irradiance response curve (IRC) was constructed showing the relationship between the normalised minimal pupil size and intensity (Fig.6). At the highest light intensity used we attained a normalised minimum pupil size of $0.09 \pm 0.001$ (mean \pm SEM, $n=5$ ), approximate $>90 \%$ constriction. The amount of constriction declined as a function of light intensity, with the dimmest light invoking the least response.

Table 1. Effective quantal flux density at different wavelengths and illuminating power levels

\begin{tabular}{|c|c|c|c|c|}
\hline \multirow{3}{*}{$\begin{array}{c}\text { Power per } \\
\text { wavelength } \\
(\mu \mathrm{W})\end{array}$} & \multicolumn{4}{|c|}{$\begin{array}{c}\text { Quantum flux density } \\
\left(\log _{10} \text { effective photons } / \mathrm{cm}^{2} / \mathrm{s}\right)\end{array}$} \\
\hline & \multicolumn{3}{|c|}{ Dual-wavelength SCWL } & Xe Arc \\
\hline & $560 \mathrm{~nm}$ & $480 \mathrm{~nm}$ & $480+560 \mathrm{~nm}$ & $480 \mathrm{~nm}$ \\
\hline 1000 & 15.75 & 15.68 & 16.02 & - \\
\hline 100 & 14.75 & 14.68 & 15.02 & 14.51 \\
\hline 10 & 13.75 & 13.68 & 14.02 & 13.51 \\
\hline 1 & 12.75 & 12.68 & 13.02 & 12.51 \\
\hline 0.1 & 11.75 & 11.68 & 12.02 & 11.51 \\
\hline 0.01 & 10.75 & 10.68 & 11.02 & 10.51 \\
\hline
\end{tabular}

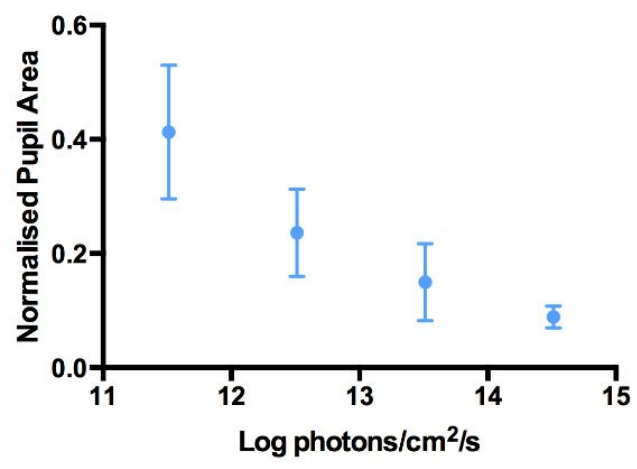

Fig. 6. The pupillary light reflex of dark-adapted mice using a $480 \mathrm{~nm}$ light generated by a xenon arc source. Irradiance response curve shows maximum pupil constriction to the brightest light, with a decline in response as intensity reduces.

The use of the SCWL source allowed us to generate two optical beams, power equivalent, of different wavelengths, 560 $\mathrm{nm}$ and $480 \mathrm{~nm}$. Following dark adaptation, a $560 \mathrm{~nm}$ stimulus was presented for 15 seconds. This allowed the pupil to reach a steady state at the high intensities of light used at this wavelength. Under these conditions, we were able to generate an approximate $60 \%$ constriction at the brightest intensity; however, this soon tapered off, as the stimulus intensity was reduced (fig. 7). Following the 15 seconds of exposure to the $560 \mathrm{~nm}$ light, we presented a second pulse consisting of $480 \mathrm{~nm}$. The combination of the $560 \mathrm{~nm}$ and the $480 \mathrm{~nm}$ stimulus was able to generate a full constriction in a similar manner to that achieved with the Xenon arc. On further analysis, the overall decrease in sensitivity is statistically significant, with a reduced sensitivity observed with the $560 \mathrm{~nm}$ light stimulus $\left(p<0.001, \mathrm{~F}_{(3,58)}=58.48\right)$. This decrease in constriction, relative to $480 \mathrm{~nm}$, can be attributed to alterations in mouse retinal photoreceptor responsiveness. Finally, both IRCs produced using the Xeon arc and SCWL (480 nm $+560 \mathrm{~nm}$ configuration) were statistically identical $\left(p>0.05, \mathrm{~F}_{(1,58)}=0.23\right.$, Fig. 8), further supporting the viability of the SCWL source in driving the PLR. 


\begin{tabular}{|c|c|c|c|c|}
\hline & \multicolumn{4}{|c|}{ Dual-wavelength with SCWL } \\
\hline $\begin{array}{c}480+560 \mathrm{~nm} \\
\text { intensity }\end{array}$ & $\begin{array}{c}16 \log \\
\text { photons } / \mathrm{cm}^{2} / \mathrm{s}\end{array}$ & $\begin{array}{c}15 \log \\
\text { photons } / \mathrm{cm}^{2} / \mathrm{s}\end{array}$ & $\begin{array}{c}14 \log \\
\text { photons } / \mathrm{cm}^{2} / \mathrm{s}\end{array}$ & $\begin{array}{c}13 \log \\
\text { photons } / \mathrm{cm}^{2} / \mathrm{s}\end{array}$ \\
\hline $\begin{array}{c}t=-3 \mathrm{~s} \\
\text { No light }\end{array}$ & & & & \\
\hline $\begin{array}{l}\mathrm{t}=10 \mathrm{~s} \\
560 \mathrm{~nm}\end{array}$ & & & & \\
\hline $\begin{array}{c}\mathrm{t}=20 \mathrm{~s} \\
560 \mathrm{~nm}+ \\
480 \mathrm{~nm}\end{array}$ & & & & \\
\hline & (a) & (b) & (c) & (d) \\
\hline
\end{tabular}

(e)

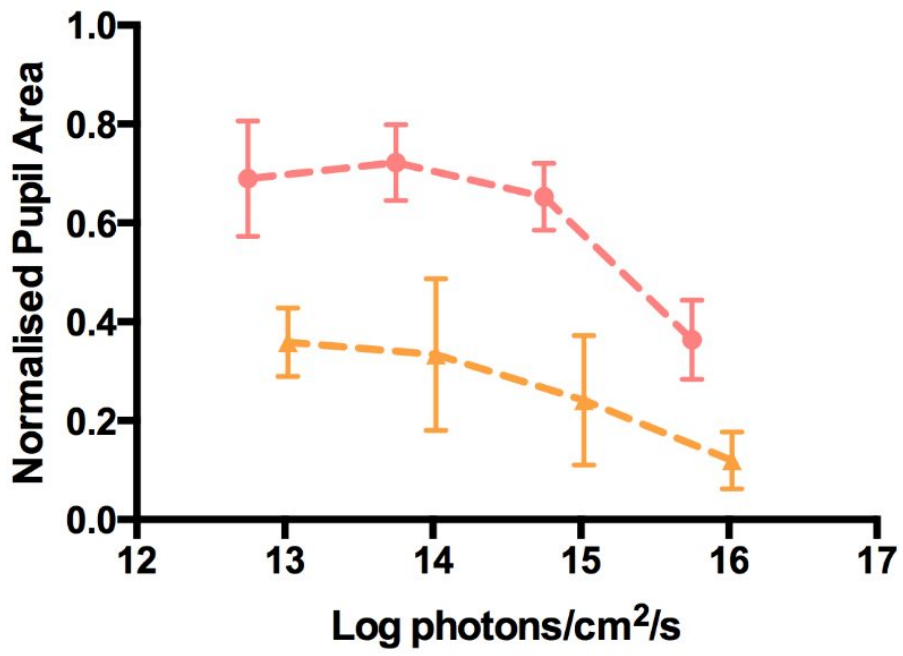

Fig. 7. (a)-(d) pupil images from dark adapted ( $\mathrm{t}=-3 \mathrm{~s})$ through to $560 \mathrm{~nm}$ light $(\mathrm{t}=10 \mathrm{~s})$ and finally $480 \mathrm{~nm}+560 \mathrm{~nm}$ ( $\mathrm{t}=20 \mathrm{~s})$. (e) Irradiance responses to a $560 \mathrm{~nm}$ light $(\mathbf{O})$ were significantly $(p<0.0001)$ less sensitive than those achieved using a $560 \mathrm{~nm}+480 \mathrm{~nm}(\boldsymbol{\Delta})$ stimul $u$ s. 


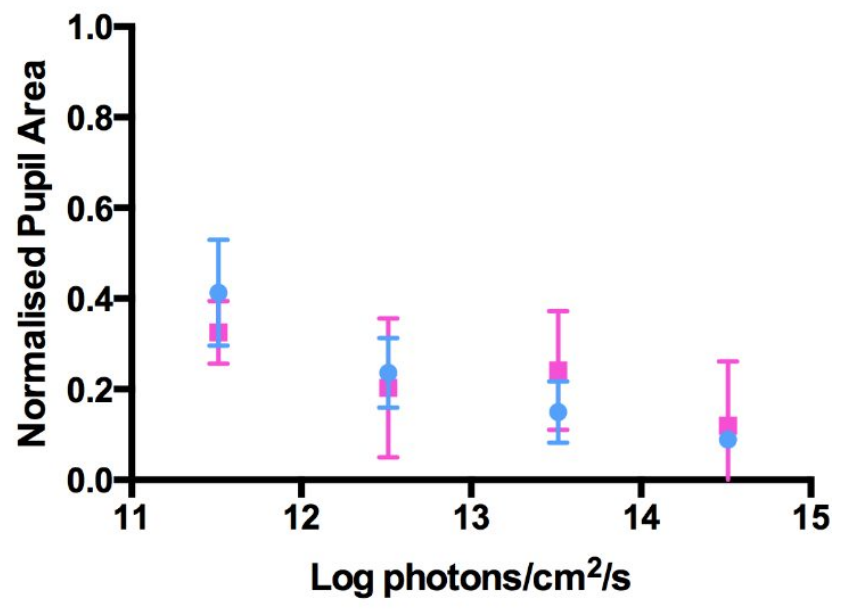

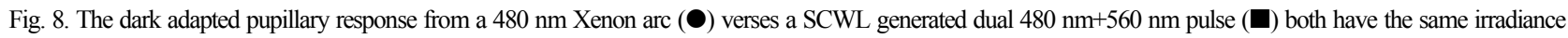
response profile $(p>0.05)$. Thus, affirming the use of the SCWL as a viable alternative to the traditional Xenon arc light source.

\section{Discussion}

The mammalian retina exhibits varying spectral sensitivity to both environmental and full spectrum white light. It is most sensitive to 'daylight' blue, something that had developed through evolution [11]. With the use of the SCWL we were able to show this shift in spectral sensitivity remarkably easily. The mouse retina was less sensitive to the $560 \mathrm{~nm}$ light; however, we were able to recover the full response by the presentation of a $480 \mathrm{~nm}$ stimulus. This difference can be attributed to the responsiveness of the photoreceptors to photic wavelengths.

The use of a SCWL is a significant leap forward from the Xenon arc light traditionally used in recording pupillary light responses. The SCWL gives the experimenter much more control over the light stimulus, through wavelength, intensity and, most importantly, a dual light configuration. Together, this will allow more complex lighting protocols to be developed that can further assist in unraveling the complex coding of light that gives rise to the pupil light reflex and other photic driven physiological responses [12].

The SCWL has a great advantage over other traditional bespoke systems. A typical dual-wavelength stimulus, using a Xenon arc, would require a complicated configuration of optical band pass filters, shutters and light/ power generators. This presents with some significant limitations; for instance, a two-wavelength optical configuration would require two optical pathways, thus increasing the complexity and footprint of the apparatus. Our approach minimizes space usage and eliminates the need for sequential light illumination.

One of the major issues faced with any experimental protocol using filtered light from a full spectrum light source is the consistency of power output at varying wavelengths. The use of a SCWL laser enables us to select the output power at a specific wavelength. This is possible because the power density is proportional to the laser repetition rate and the optical bandwidth; the SuperK VARIA is able to maintain these parameters constantly across the entire visible spectrum for every wavelength, thus allowing the user to select the desired power output. In addition the output intensity can be manipulated by varying, the picoseconds pulse duration and optical bandwidth in steps of nanometres, thus maximizing the available spectrum peak power.

The timing of a stimulus is also critical. We were able to use a delayed onset in light stimulus in order to highlight the spectral sensitivity of the retina to different wavelengths. Our SCWL has a variable repetition rate ranging from $2 \mathrm{MHz}$ to $80 \mathrm{MHz}$, which can generate triggering rates between $15 \mathrm{~ns}$ to $0.5 \mu \mathrm{s}$; thus allowing rapid cycling of stimuli with varying intensities and wavelengths.

There is great potential in using the SCWL in both basic biological sciences and within the clinical setting. We have just touched the surface here by demonstrating the usefulness of a dual wavelength light source in dissecting out physiological spectral sensitivity in a mouse model. In addition, there is a range of clinical uses for such a system spanning from neurosurgery, psychiatry, and ophthalmology through to sleep medicine $[9,10,13]$. There is a wide 
spectrum of uses/ application for the SCWL and we believe this to be of great benefit to the advancement of science and healthcare.

\section{Funding.}

UBAPHODESA Marie Curie European Industrial Doctorate (607627); ERC COGATIMABIO (249889), Royal Society Wolfson Research Merit Award; Moorfields Eye Hospital NHS Foundation Trust; UCL Institute of Ophthalmology.

\section{Acknowledgment.}

We thank Charles River laboratories, Margate for their support. We would also like to extend our thanks to Min Keating for assisting in constructing our schematic diagrams.

\section{References}

1. R. J. Lucas, G. S. Lall, A. E. Allen, and T. M. Brown, "How rod, cone, and melanopsin photoreceptors come together to enlighten the mammalian circadian clock," Progress in brain research 199, 1-18 (2012).

2. D. G. McMahon, P. M. Iuvone, and G. Tosini, "Circadian organization of the mammalian retina: from gene regulation to physiology and diseases," Progress in retinal and eye research 39, 58-76 (2014).

3. C. M. Altimus, A. D. Guler, N. M. Alam, A. C. Arman, G. T. Prusky, A. P. Sampath, and S. Hattar, "Rod photoreceptors drive circadian photoentrainment across a wide range of light intensities," Nature neuroscience 13, 1107-1112 (2010).

4. G. S. Lall, V. L. Revell, H. Momiji, J. Al Enezi, C. M. Altimus, A. D. Guler, C. Aguilar, M. A. Cameron, S. Allender, M. W. Hankins, and R. J. Lucas, "Distinct contributions of rod, cone, and melanopsin photoreceptors to encoding irradiance," Neuron 66, 417-428 (2010).

5. R. J. Lucas, R. H. Douglas, and R. G. Foster, "Characterization of an ocular photopigment capable of driving pupillary constriction in mice," Nature neuroscience 4, 621-626 (2001).

6. R. J. Lucas and R. G. Foster, "Neither functional rod photoreceptors nor rod or cone outer segments are required for the photic inhibition of pineal melatonin," Endocrinology 140, 1520-1524 (1999).

7. D. Lupi, H. M. Cooper, A. Froehlich, L. Standford, M. A. McCall, and R. G. Foster, "Transgenic ablation of rod photoreceptors alters the circadian phenotype of mice," Neuroscience 89, 363-374 (1999).

8. R. J. Lucas, S. Hattar, M. Takao, D. M. Berson, R. G. Foster, and K. W. Yau, "Diminished pupillary light reflex at high irradiances in melanopsin-knockout mice," Science 299, 245-247 (2003).

9. A. E. Neice, M. Behrends, M. P. Bokoch, K. M. Seligman, N. M. Conrad, and M. D. Larson, "Prediction of Opioid Analgesic Efficacy by Measurement of Pupillary Unrest," Anesthesia and analgesia (2016).

10. A. C. Lukaszewicz, D. Dereu, E. Gayat, and D. Payen, "The relevance of pupillometry for evaluation of analgesia before noxious procedures in the intensive care unit," Anesthesia and analgesia 120, 1297-1300 (2015).

11. T. M. Brown, "Using light to tell the time of day: sensory coding in the mammalian circadian visual network," The Journal of experimental biology 219, 1779-1792 (2016).

12. B. Feigl and A. J. Zele, "Melanopsin-expressing intrinsically photosensitive retinal ganglion cells in retinal disease," Optometry and vision science : official publication of the American Academy of Optometry 91, 894-903 (2014). 